\title{
Tangence
}

\section{La référence comme effet de lecture}

\section{Max Roy}

Numéro 44, juin 1994

La référence littéraire

URI : https://id.erudit.org/iderudit/025814ar

DOI : https://doi.org/10.7202/025814ar

Aller au sommaire du numéro

Éditeur(s)

Tangence

ISSN

0226-9554 (imprimé)

1710-0305 (numérique)

Découvrir la revue

Citer cet article

Roy, M. (1994). La référence comme effet de lecture. Tangence, (44), 66-80. https://doi.org/10.7202/025814ar d'utilisation que vous pouvez consulter en ligne.

https://apropos.erudit.org/fr/usagers/politique-dutilisation/ 


\section{La référence comme effet de lecture Max Roy}

La lecture n'est jamais exclusivement littérale ou référentielle. Elle est dynamique et elle convoque plusieurs attitudes, plusieurs dimensions du texte à la fois, qui s'éclairent ou s'enrichissent mutuellement. Il se produit, en fait, un aller-retour de la lecture littérale et référentielle en fonction d'une recherche de sens. La distinction n'est pas marquée matériellement, mais elle est opératoire dans l'activité de lecture. Ainsi, et cela paraît un truisme, lorsqu'il interprète sémiotiquement un élément du texte en fonction de sa valeur syntaxique ou lexicale manifeste, le lecteur porte attention à sa dimension littérale. Lorsqu'il interprète sémantiquement le texte à la lumière des faits extérieurs, de l'Histoire sociale, par exemple, son attention est tournée vers une dimension référentielle. Toutefois, la généralité de cette observation introduit une confusion qu'il faut lever ici.

Pour parler de la référence littéraire comme effet de lecture, on peut adopter au moins deux hypothèses élémentaires. La première veut que le texte impose ses références; la seconde postule que la lecture cherche à construire sa référence, ses points d'appui et de repère. S'il y a une part d'identification dans chaque acte de lecture, il est bien difficile d'en rechercher les motivations sans verser dans le psychologisme ou dans le sociologisme. Certes, il existe des conditions objectives, inscrites dans les textes littéraires, d'une lecture référentielle. Mais les effets de réel sont bien autre chose que les référents virtuels de signes textuels. Peut-on isoler une forme de lecture en fonction de cet aspect?

En pratique, le référentiel est indissociable du signe littéraire. Qu'est-ce qui pourrait y échapper? Le jamais-vu, l'inédit? Pour combien de temps? La modernité n'a-t-elle pas aussi ses références? Si tout fait référence dans le texte littéraire, alors la littérature et la référence sont une seule et même chose. La position inverse n'est pas plus défendable, car une lecture référentielle ne fait pas pour autant abstraction de la littérature. Elle n'est pas une échappée ou un refus mais plutôt un investissement du sujet, l'un de ses modes d'adhésion, le plus courant sans doute sinon le plus immédiat. Nous savons bien, par ailleurs, que le sens n'est pas 
réductible à la référence. Et lorsqu'il s'y trouve lié, de quel ordre est cette relation? S'agit-il d'une valeur surajoutée? Est-ce le résultat d'intentions convergentes ${ }^{1}$ ? N'est-ce pas couramment un effet de cohérence interne? Ce sens anaphorique est bien près du sens minimal. Or, le lisible, comme l'intelligible, ne se réduit pas à la sémantique. À la limite, tout "fait sens " pour le lecteur.

\section{Un seuil de compétence}

Il est impossible de traiter de la lecture sans faire intervenir le lecteur. Pourtant, la lecture s'évanouit sitôt achevée, même lorsqu'elle produit des effets durables chez le lecteur. Elle est une opération dont le résultat inconstant ne laisse pas de traces matérielles et analysables. Cela n'interdit pas d'examiner la question en rapport avec la référence littéraire d'un point de vue théorique essentiellement ${ }^{2}$.

À la condition d'être compétente, bien sûr, la lecture du texte littéraire est tout à la fois une perception de la lettre et de son audelà. Hormis les aptitudes de base, de multiples facteurs externes interviennent dans la compétence de lecture et dans sa pratique.

1 Je renvoie aux trois types d'intentions prises en compte dans les études herméneutiques, soit l'intentio auctoris, l'intentio operis et l'intentio lectoris, et que Umberto Eco inclut dans sa réflexion sur l'interprétation. Voir " Intentio lectoris". Notes sur la sémiotique de la réception", dans Les limites de l'interprétation, Paris, Grasset, 1992.

2 L'étude de cette question conduit à imaginer l'élaboration d'un parcours interprétatif. S'il s'agit d'un modèle virtuel, sa réalisation n'est pas moins abstraite. Seule sa vérification par mode d'enquête, qui constituerait un retour sur la pratique, pourrait avoir un caractère concret. Mais elle comporterait aussi les inconvênients d'une distance établie par la conscience à l'égard de l'action. La réponse serait une construction discursive. À défaut d'un accès à la conscience de la lecture, on peut s'en remettre à des lectures attestées par la critique et transformées parfois en programmes de lecture pour identifier des schèmes et des effets d'interprétation. J'en ai fait l'objet d'une communication intitulée: :Rééditions et relectures. Éléments d'une histoire de la lecture littéraire, au Québec*, lors du colloque "L'acte de lecture *, tenu à Québec en 1992 (ouvrage à paraître, sous la direction de Denis Saint-Jacques et alli). Ce genre de lectures fait intervenir une médiation importante, de l'ordre de la sanction sociale et culturelle, qui poursuit dans sa visée persuasive une pratique réglée. Ia critique scolaire, en l'occurrence, enseigne comment lire; elle initie la lecture, la prépare, la dirige même. Quant à la critique d'humeur ou à la critique spécialisée, leurs fins ne font pas moins écran à la lecture qu'elles supposent mais dont elles s'écartent pour produire des constructions signifiantes. 
68

Hors l'espace du texte, la situation de l'œuvre n'est pas négligeable. On sait ce qu'il en a coûté à des écrivains que leur œuvre ait été lue dans la perspective d'une référence directe au contexte politique ou idéologique. On admettra sans peine, par exemple, limportance du contexte qui a accompagné la parution des Versets sataniques. Absolument déterminante sur la diffusion et sur la réception de l'œuvre, on peut présumer qu'une telle situation a également des conséquences sur la lecture, sur sa motivation et sur son parcours, quoique cette influence reste impossible à mesurer. Il suffit d'examiner l'accueil de la critique et les relectures d'œuvres censurées ou condamnées pour des raisons idéologiques pour constater qu'un sort extérieur leur reste inévitablement attaché, comme s'il faisait partie de l'œuvre et devait être lu avec elle, dans la transparence du texte ${ }^{3}$.

\section{L'espace du texte}

D'un point de vue formaliste, le contexte de diffusion d'une œuvre est sans pertinence. Même en considérant la dimension implicite des énoncés, la référence se réduit alors au domaine discursif. Elle y est comme entièrement inscrite, ce qui confine la question de la référence à celle des contenus littéraires et de leur manifestation. Sans aller jusqu'à "l'imitation de la nature" ou jusqu'à l'indissociabilité de l'expression et du contenu, on admettra que la fonction de représentation n'est pas réservée à une sémantique de la langue pas plus que les objets d'un réel empirique ne constituent tout le représentable. En pratique, la représentativité devient un facteur parmi d'autres de la lisibilité d'une ouvre littéraire. Pour en rester à l'espace du texte, convenons de la pertinence des phénomènes de forme ou d'expression en général. Comme l'esprit humain n'est pas une machine, il ne se contente pas d'enregistrer et de relier des faits; il en est éventuellement transformé d'un point de vue intellectuel, affectif ou même physique. Au-delà des automatismes que décrivent les opérations cognitives, la compréhension des formes d'expression passe par leur déconstruction, mais leur interprétation appelle bien plus

3 En dehors d'une coopération textuelle, il existe une coopération littéraire qui se manifeste notamment par la circulation des propositions, arguments et jugements sur les ouvres. A une autre occasion, j'ai appelé cette interprétation itérative un " parcours interlectoral *. 
que la reconnaissance de leur fonction syntaxique; elle les investit d'intentions.

À l'instar des matériaux du peintre, des tons et des textures, il est certain que tous les faits de discours sont significatifs. Ils n'échappent pas eux-mêmes à un contexte d'intellection, que la critique pourra rendre explicite. Ainsi, les symétries, équivalences ou différences d'expression produisent des effets de sens reconnaissables, dont celui de mettre en évidence (en valeur) certains contenus mais également celui de signaler une organisation, une construction, de marquer l'appartenance du texte à un courant, à un genre, à une sorte de modèle, bref à une référence de convention ou de tradition. La recherche d'une isomorphie, d'un mimétisme ou d'une valeur opératoire dans la prosodie, dans les phénomènes d'allitération ou de rythme n'est-elle pas, par delà la réponse à une volonté de compréhension, un énoncé de cohérence exigée ou de référence présupposée? Ces exigences et ces présuppositions appartiennent à la culture littéraire et, singulièrement, à la culture scolaire. La valeur des sonorités dans un poème, dont on présume qu'elles doivent aller de pair avec le sujet pour produire une impression d'ensemble, n'est pertinente qu'en fonction de la référence interne. Hormis les conditions de crédibilité de l'interprète, c'est sur la base de cette argumentation, par exemple, que les consonnes $m$ ou $p$ dans un vers de Lamartine peuvent évoquer la langueur. C'est par une autre référence, externe, qu'on en jugerait si l'expression et le contenu paraissaient "mal assortis". On s'en remettrait au code culturel et à l'appréciation critique. Mais des jugements aussi opposés dans leur principe signalent, en fait, un même horizon épistémique: un discours académique sur la poésie. Leur référence appartient à une tradition.

\section{La tradition littéraire}

Les rapports entre la lecture, la référence et la littérature peuvent être considérés sous cet angle particulier. Ainsi, la lecture littéraire de la référence suppose un certain mode de compréhension et d'interprétation. Ce doit être une lecture appropriée à l'œuvre littéraire ${ }^{4}$. Même dans une perspective très large, allant

4 Voir, à ce propos, une livraison précédente de la revue Tangence consacrée à "la lecture littéraire* ( ${ }^{\circ} 36$, mai 1992). 
de l'histoire à la sémiotique en passant par une sociologie de la culture, on peut observer que cette pratique spécialisée est déterminante. Elle intervient, en effet, dans la caractérisation des textes promus au rang d'œuvres littéraires, dans la consécration d'un corpus en expansion et dans une définition même de la littérature.

Ces effets de valorisation littéraire ${ }^{5}$, ultime conséquence des jugements critiques, débordent la question de la référence et la déportent dans le monde des vérités et des moralités. À ce compte, les interrogations sur la vraisemblance des contenus discursifs ou narratifs relèvent également d'une sanction de vérité ou d'une approbation morale, dans lesquelles elles trouvent une résolution. L'acceptabilité des contenus ne se suffit pas d'une adéquation ou d'une présomption de fidélité au réel. La recherche de la référence, pas plus que l'illusion réaliste, ne saurait être confondue avec l'objectivité qui n'est souvent qu'une caution rationnelle. Une activité aussi variable et aléatoire que la lecture y trouve une possible garantie de compréhension mais non des règles d'exécution ni même une finalité. Dans la critique, en l'occurrence, les énoncés sur la vraisemblance littéraire s'avèrent souvent des jugements sur le goût que l'argumentation ne parvient pas à camoufler. La contestation des références dans un texte est aussi bien alors un aveu de préférence.

Prenons le cas d'une ouvre québécoise qui, depuis plus de cinquante ans, s'est mérité tous les éloges et que la problématique de la référence appelle presque inévitablement. Il s'agit du roman Trente arpents, de Ringuet ${ }^{6}$, qui est devenu dans le discours de l'histoire littéraire l'un des meilleurs représentants du "roman de la terre ". À première vue, la cause est entendue: la référence est explicite et sa signification, transparente. Le roman a

5 Je me suis longuement intéressé à ces questions en rapport avec l'enseignement de la littérature dans les collèges classiques du Québec au $x_{x} x^{e}$ et au $\mathrm{xx}^{\mathrm{e}}$ siècle ("Le discours didactique: un lieu inaugural des valeurs littéraires ", thèse de doctorat, Faculté des lettres, Université Laval, 1991, 494 f.). Voir en particulier: "La valorisation cognitive des objets littéraires ", dans La lecture et ses traditions, sous la direction de Joseph Melançon, Nicole Fortin et Georges Desmeules, Québec, Nuit blanche éditeur, sous presse.

6 Paris, Flammarion, 1938. Je renvoie ici à l'édition critique préparée par Jean Panneton avec la collaboration de Roméo Arbour et Jean-Louis Major: Ringuet, Trente appents, Montréal, Les Presses de l'Université de Montréal, «Bibliothèque du Nouveau Monde *, 1991. 
maintes fois été qualifié d'œuvre réaliste ou naturaliste même si l'auteur se défendait bien de faire dans ce genre en écrivant: "Ce livre n'est pas un roman "régionaliste"; les paysans que j'ai connus n'étaient pas des héros. Ce livre n'est pas un roman "naturaliste"; les paysans que j'ai connus n'étaient pas des brutes " 7 . Loin d'être la copie conforme d'un modèle idyllique, comme celui qu'avait imaginé Damase Potvin ${ }^{8}$, Trente arpents renferme une dimension critique certaine. La vision qu'il propose de la vie paysanne s'accorde mal avec la propagande agriculturiste. Pour cette raison, du reste, le roman s'est attiré quelques critiques négatives, ce qui n'a pas empêché qu'il fut acclamé et largement diffusé 9 . On désapprouvait alors son pessimisme.

\section{La langue imaginaire}

Les réserves de certains commentateurs affichent nettement leurs présupposés lorsqu'elles concernent les questions de langue. Comme on le sait, Trente arpents invite à lire des langages contrastés, l'auteur ayant eu recours à des niveaux de langue distincts dans la narration et dans les dialogues, ce qui se rattache à une tradition romanesque séculaire. À une langue très précise, recherchée même, s'oppose un patois campagnard. Les accents de l'oralité y accompagnent un vocabulaire marqué. À dessein de produire la vraisemblance, l'usage de l'expression familière va parfois jusqu'à l'irrévérence. Le vieux paysan Euchariste Moisan lui-même ne peut se retenir de sacrer pour exprimer sa révolte ou sa douleur. Ces rares "écarts de langage", comme l'on disait alors, passent à peu près inaperçus de nos jours. Ils ont valu à l'auteur du roman les reproches que l'on imagine, fondés sur l'argument suivant: un profond sentiment religieux étant le lot des habitants de la campagne, le vieux paysan ne pouvait tout simplement pas se comporter ainsi. Cela était invraisemblable, car contraire à l'opinion que l'on se faisait des moeurs rurales, contraire à leur indissociable moralité ${ }^{10}$. C'était l'inverse du bon sens.

7 Ibid., p. 16.

8 Restons cbez nous!, Québec, J.-Alfred Guay, [1908], 243 p.

9 Le fait d'avoir été édité par Flammarion, une prestigieuse maison française, n'est sans doute pas étranger au succès du roman et peut-être aussi au peu d'influence de ses détracteurs.

10 Dans un autre contexte, des arguments tout aussi désapprobateurs ont été invoqués à propos des œuvres publiées chez l'éditeur québécois Parti pris 
72

La référence souhaitée, à partir de laquelle on jugeait de l'invraisemblance, était une opinion commune. C'était une référence imaginaire.

Cette référence imaginaire était une conception de lecteur avant d'être une conception de critique. Tout lecteur se fait une idée plus ou moins juste ou élaborée de ce à quoi renvoie un texte, idée qui se développe en se confirmant au fil de sa lecture. Cette référence imaginaire s'élabore à partir des signaux du texte, du lexique et de ses usages connus mais aussi, évidemment, à partir des lectures antérieures, des habiletés acquises par l'expérience ou l'étude et des sources extérieures. Des conceptions culturelles datées font partie, le cas échéant, des références communes à retenir dans l'examen de l'activité de lecture. Il en va ainsi, par exemple, des idées répandues à l'égard du Nouveau monde et de leurs représentations littéraires, dans les siècles passés. De telles références font partie du contexte de lecture à une époque donnée. Elles s'ajoutent éventuellement aux références historiques d'un lecteur qui a le privilège de la postérité. Paradoxalement, il faut tenir compte de cette part inévitable d'imaginaire dans la référence que se construit le lecteur — ou qui se construit à son insu - pour envisager son rapport au texte et sa capacité d'y "lire " des références.

Le roman de Ringuet ne se soustrait pas à cette exigence sous prétexte que le temps, l'espace et la situation qu'il décrit étaient bien connus de ses contemporains ${ }^{11}$. Bien qu'il ait toutes les apparences d'une réalité familière, il en appelle à d'autres facteurs pour imposer sa référence et devenir un "classique", c'est-à-dire une référence incontournable. Par exemple, la succession naturelle des événements, la division du roman correspondant aux quatre saisons de la vie d'Euchariste Moisan, reproduit une structure courante. Cette conformité à un usage romanesque signale la tradition littéraire. Même pour les lecteurs non avisés, sa significa-

durant les années 1960 . Le scandale du joual a débordé l'espace de la critique, conduisant parfois à la censure, et l'enseignement de ces œuvres a même été sévèrement sanctionné.

11 Au fait, la datation est imprécise et c'est une recherche minutieuse qui permet d'établir les limites temporelles de l'action, depuis le premier jusqu'au dernier épisode du roman, soit de 1887 à 1932. Quant au décor, de l'avis des auteurs de la récente édition critique (collection BNM), il correspondrait aux environs de Maskinongé, à quelque cinquante kilomètres de Trois-Rivières, alors que les noms de lieux dans le roman sont imaginaires. 
tion est univoque. C'est dans l'ordre! Il s'agit aussi d'un schème culturel : les quatre saisons représentent les âges de la vie. La métaphore est devenue cliché. Pour décrire une tradition de pensée et d'action en déclin, l'auteur s'en remet à une forme connue qui assure la vérité de l'ensemble. Ce qui était inadmissible dans une idéologie conservatrice encore très influente - quoique mal adaptée à la situation socio-économique - était parfaitement recevable dans une perspective littéráire. Par sa structure, l'histoire était parfaitement crédible et la thèse qu'elle pouvait sous-tendre, sa conclusion pessimiste, était également valable.

\section{La croyance partagée}

La crédibilité des énoncés met en cause l'énonciation, mais également la qualification et la compréhension progressive du lecteur. À ce titre, les croyances comptent au moins tout autant que les connaissances objectives. Faisant appel à ses références antérieures pour identifier les propositions du texte, le lecteur actuel d'un roman comme Trente arpents se voit obligé d'admettre - non d'approuver - une hiérarchie de valeurs, des caractéristiques de pensée et de langage ainsi que des convictions populaires qui paraissent dépassées. Un exemple suffira :

Le printemps était arrivé, celui du calendrier, bientôt suivi de celui de la nature, un printemps hâtif annoncé par tous les signes habituels et qui ne trompent pas. Il était encore trop tôt pour l'arrivée des premières corneilles, mais on avait vu un ours; et tout un chacun sait que les ours sortent le 25 mars et ne rentrent point $s$ 'ils voient leur ombre; ce qui est le signe d'un printemps tiède et court. ${ }^{12}$

On peut soupçonner une certaine ironie dans ce passage qui fait appel à la sagesse populaire. Ce n'est pas un discours de conviction, mais un énoncé d'évidence. La formule rhétorique de cette évidence est la généralisation, qui rapproche le dicton du proverbe. L'énoncé de savoir entraîne le lecteur à admettre au moins un mode de pensée. Il fait apparaître une croyance transformée en "science". Du point de vue de l'interprétation, le dicton, le proverbe et le cliché sont des indications d'une façon de lire. Ils invitent le lecteur à adapter son interprétation à l'univers interne de l'œuvre, soit à la manière dont se déroulent les événements et 
dont vivent les personnages, en fonction d'un corps de croyances et de connaissances. Dans le cas présent, l'évidence proverbiale rappelle un ordre du monde fondé sur des certitudes, sur les lois de la nature, en l'occurrence, ce qui sous-entend l'idée d'un déterminisme. Bien sûr, il importe peu que le lecteur connaisse ou ignore la date du réveil des ours. Qu'il lui suffise de se reporter au contexte dans lequel ce savoir avait quelque valeur pour $y$ trouver matière à sourire ou à s'émouvoir.

\section{Le fait d'histoire}

À côté de cela, le fait d'histoire paraît une référence beaucoup plus sûre, plus objective et, partant, plus facilement lisible et interprétable. Parmi les événements historiques évoqués dans le roman de Ringuet, signalons la Première Guerre mondiale (1914-1918) et la grande crise économique des années 1930 dont tout lecteur a au moins entendu parler. Ils sont considérés essentiellement dans le contexte québécois. Le début de la partie intitulée "l'automne" coïncide précisément avec la période de la conscription. Quant à "l'hiver", saison d'un funeste exil, il est associé à la crise. Retenons le premier événement qui donne lieu à une scène de bravoure verbale et au commentaire explicatif suivant :

Des élections générales eurent lieu, où sans que la loi de conscription fût nettement mentionnée, tout le monde la savait dans l'air. Et ce fut la division nette du pays, l'éclatement brusque de cette ombre de lien qui retenait ensemble le Canada français et l'anglais. Dans le Québec on crut tous les soldats, à qui droit de vote avait été donné, tous les "Anglais*, dont les fils étaient déjà partis, ligués pour forcer les récalcitrants. La province fit bloc.

En vain. Les Chambres, dès leur réunion, votèrent la loi; mais l'exemption des fils de paysans et, plus encore, l'absence de chefs empêchèrent le mouvement de révolte de prendre corps et de s'étendre. Pour un peu, à cent ans d'intervalle, '37 se fût répété. Il y eut à Montréal quelques assemblées violentes; Québec connut même l'émeute ouverte. Mais tout s'éteignit vite.

Cela suffit cependant pour que tous les profiteurs de la guerre, officiers de parade, aspirants ministres, munitionnaires et fournisseurs de l'armée criassent à la lâcheté. Quand la parole de Bourassa se fut soumise et que l'épiscopat fidèle à sa politique centenaire eut requis l'obéissance, il ne se trouva plus de voix qui fît connaître et expliquât à la fois le refus de tout un petit 
peuple brisé. Personne pour dire en leur nom combien simple était tout cela. ${ }^{13}$

Pour qui ne connaît pas l'histoire du Québec, ces extraits comportent au moins deux embûches. Dans le dernier paragraphe, le nom propre pose une difficulté qui ne tient pas longtemps. Dès que le contexte de lecture et le contexte de l'histoire sont distingués, comme il se doit, il tombe sous le sens qu'il ne peut pas s'agir d'un homme politique encore actif récemment. Mais de qui s'agit-il? Les lecteurs avertis auront reconnu le fondateur du Devoir, Henri Bourassa, qui fut aussi député libéral et fervent nationaliste. Mais les autres n'auront pas trouvé dans le texte réponse à leurs interrogations.

Une difficulté différente apparaît dans le paragraphe précédent avec le passage: "Pour un peu, à cent ans d'intervalle, '37 se fût répété " où il est fait référence à l'insurrection des Patriotes canadiens, aussi appelée la Rébellion de 1837-1838. Pour être comprise, l'allusion exige des connaissances historiques assez précises et, pour être envisageable, la perspective d'une répétition de ces événements suppose une familiarité avec un contexte socio-politique. J'ai pu constater, sur ce point précis, une fréquente confusion chez des lecteurs en milieu scolaire qui, croyant lire 1937 au lieu de 1837, ont fait un rapprochement avec la deuxième grande guerre. Une dimension socio-politique de l'œuvre leur a ainsi totalement échappé à cette occasion ${ }^{14}$. Chez certains lecteurs, la perception de la chronologie romanesque en a été transformée. Mais leur lecture n'a pas été impossible du fait de ne pas avoir actualisé un programme de signification, en tout cas pas le programme prévisible. Il est plutôt remarquable qu'elle ait cherché une cohérence, au point de confondre des dates (1837, 1937 ou même 1939) et d'ignorer l'indication "à cent ans d'intervalle . C'est à supposer qu'une telle cohérence, provisoire et rectifiable, est nécessaire à la poursuite de la lecture, dont la démarche se trouve ainsi validée. Tributaire d'une faute d'attention ou d'un manque d'information historique, cette cohérence sans fondement référentiel serait néanmoins fonctionnelle dans l'économie de l'activité de lecture. On pourrait parler alors d'une "illusion cognitive" à la manière de

13 Ibid., p. 292-293.

14 .En fait, il est question de l'élection de Robert Borden en 1917 et de la crise de la conscription. 
76

Bertrand Gervais ${ }^{15}$. Le fait d'histoire ne garantit pas une lecture univoque ou "orthodoxe", mais il accrédite des certitudes.

\section{La voie allégorique}

On peut penser que ces questions trouvent leur pertinence dans la perspective de la référence en raison du genre de roman ici en cause. Bien entendu, la référence se découvre, se lit aussi sur le mode allégorique. Nous en faisons l'expérience quotidienne dans le langage figuré et dans le jeu de mots. Dans une esthétique non réaliste, c'est-à-dire où n'est pas centrale la représentation d'un environnement extérieur, les effets de référence littéraire ne sont pas absents, du simple fait d'abord de la référence linguistique - la relation entre un signe et un référent - , mais également en raison de la cohérence interne du discours et de son appartenance éventuelle à un genre ou à une série littéraire. Les mondes possibles des fables, romans fantastiques et textes surréalistes s'inspirent des modes d'existence réels. Bien qu'ils déforment la réalité, ils en reproduisent les rapports de consécution et les relations causales. Ce qu'ils donnent à voir, ils le donnent à croire, même si c'est pour s'en amuser ou s'en méfier. Les références internes des œuvres appartiennent même, le cas échéant, à un fonds commun. Pour s'en tenir à un genre typique, par exemple, les caractéristiques des loups-garous et des vampires constituent des références connues et difficilement contournables, fût-ce dans la parodie. Mais nous sommes bien loin alors de la référence sociale qui est, depuis plusieurs décennies, au centre de la réflexion et de la critique sur la dimension représentative de la littérature.

La critique sociale n'est pas le propre du roman réaliste. Elle est encore possible, parfois plus subversive, dans la fable ou le conte. Les dimensions socio-politiques des contes d'un Jacques Ferron, par exemple, sont bien connues. Dans Les têtes à Papineau, de Jacques Godbout, l'allégorie politique est pour le moins évidente $^{16}$. Venu d'un autre horizon, le "conte orwellien" de Nina Berberova $\dot{A}$ la mémoire de Scbliemann ne manque pas de suggérer, dans un dédale de routes et de villes inconnues, des faits

15 Bertrand Gervais, À l'écoute de la lecture, Montréal, VLB éditeur, coll. "Essais critiques", 1993, en particulier p. 67-80.

16 Jacques Godbout, Les têtes à Papineau, Paris, Seuil, 1981. 
de civilisation ${ }^{17}$. Évidemment, le mode allégorique cultive la référence interne. De même que pour toute la littérature, son matériau premier est le langage. C'est peut-être aussi bien la source que l'aboutissement du texte allégorique, la condition de départ et la condition d'arrivée de la lecture.

Pour faire intervenir un dernier exemple, également bien connu, je retiens un roman de Jacques Poulin: Les grandes marées ${ }^{18}$. Sans dénier son caractère humoristique, il peut être reçu comme une forme d'allégorie sociale. Mis à part les nombreuses références qu'on peut y découvrir, quant à la géographie, la société ou la culture, il contient de multiples interrogations sur le langage et la représentation. En effet, la précision linguistique constitue une véritable obsession pour le personnage central qui est un traducteur de bandes dessinées. Ce dernier trait n'est pas insignifiant puisqu'il nous entraîne dans une aventure qui est celle de la langue et de l'image.

Considérons un épisode en particulier. Il met en scène un professeur de la Sorbonne nommé Mocassin. C'est un spécialiste de l'image, incluant la bande dessinée, qui parle d'abondance et savamment. Il ressemble à Tournesol, dans Tintin, et il est pareillement dur d'oreille. Pour corriger ce fait, on lui offre un appareil d'un nouveau genre qui consiste en une petite bille auditive. Une fois l'appareil introduit dans le conduit auditif de l'oreille du professeur, il se produit "un son étrange qui ressembl[e] à un roulement mécanique ${ }^{19}$. La suite n'est pas moins étonnante:

Mocassin, les yeux clos, dodelinait de la tête.

Un instant plus tard, le bruit de roulement fut interrompu par un timbre clair, puis il reprit et fut ensuite coupé par un cliquetis, et, pendant une minute, tous ceux qui étaient là entendirent distinctement cette série de sons : roulement... timbre... roulement... cliquetis... roulement...

- C'est comme une machine à boules, murmura l'Homme Ordinaire qui exprimait ainsi l'avis unanime des spectateurs.

Le roulement mécanique s'éteignit sur un dernier cliquetis.

17 Nina Berberova, À la mémoire de Scbliemann, Paris, Actes Sud, 1991.

18 Jacques Poulin, Les grandes marées, Montréal, Leméac, 1978.

19 Ibid., p. 133. 
78

Dans le silence qui suivit, le professeur Mocassin se mit à vaciller sur sa chaise et il tomba de tout son long sur le sol.

Il était sans connaissance. ${ }^{20}$

Une lecture réaliste de ce passage est peu indiquée. Il y a bien une référence lisible, mais qui s'apparente au monde de la bande dessinée où le burlesque, l'exagération et la caricature vont de soi. La lecture est engagée par ce mode d'expression et la compréhension passe par une série d'associations sémantiques. Assumons le risque de verbaliser l'implicite: l'appareil auditif est une bille introduite dans la tête du professeur qui en perd la boule. À cet égard, le bruit de la machine à boules vient signaler soit un jeu en action soit un dérèglement, comme si la petite boule s'égarait dans la "mécanique" du cerveau. Alors qu'il est sur le point d'avoir pleinement accès à la réalité extérieure, dans son environnement sonore du moins, le savant professeur est transformé. Pour employer une expression familière, il devient "maboul». Son évanouissement est aussi, au sens propre, une perte de connaissance.

Dans ce cas, la lecture du jeu de langage est tout à fait pertinente, à mon avis, ce qui n'en fait pas la seule interprétation possible ou correcte. Néanmoins, la suite du roman valide ce mode de lecture, notamment par le fait que le professeur, revenu à lui, n'est plus tout à fait le même. Il s'intéresse désormais aux profondeurs de l'inconscient humain et, lorsqu'il est lui-même inconscient, il adopte un pseudo-langage de primates, qui n'est autre que la langue singe imaginée par le créateur de Tarzan. L'univers fictif de la bande dessinée est signalé à nouveau dans ce passage et à maintes occasions dans le roman, conduisant le lecteur à le "prendre à la lettre", c'est-à-dire à traiter de façon particulière les signes et les référents du texte. À l'évidence, sa dimension littérale est aussi signifiante que sa dimension référentielle.

\section{Des attitudes de lecture}

Ces deux dimensions ne sont pas des données objectives, mais des objets construits. La lecture ne se superpose pas avec neutralité sur un texte, dans lequel elle trouverait son principe et son fonctionnement. Le texte ne peut prévoir tous ses usages même s'il est bien forcé de s'y plier. À l'égard de l'œuvre litté- 
raire, il existe une attitude de lecture préalable et qui, dans le pire des cas, est un désaveu ou une offense. Mis à part les détournements par une lecture utilitaire, partiale ou incriminante, il est possible d'établir des attitudes intellectuelles susceptibles d'apparaître dans toute démarche de lecture et qui font voir le caractère tout relatif de sa dimension référentielle.

Une attitude importante dans une démarche de lecture me paraît être l'analyse, entendue dans son acception la plus large et non dans celle spécialisée d'un contexte scolaire ou scientifique. Elle est paradoxalement une attitude élémentaire, fût-elle spontanée. En effet, la perception du sens lexical d'un texte obéit d'abord à un déchiffrement, à une identification de sèmes. Cette perception des éléments constitutifs repose sur une analyse, c'està-dire une décomposition du tout en ses parties ${ }^{21}$. L'exige, par exemple, la valeur de l'expression "être sans connaissance" dans le roman de Poulin. Mais la dimension référentielle du texte s'y prête également. Ainsi, l'organisation des contenus, l'intrigue, la structure temporelle ne deviennent lisibles qu'une fois comprises en elles-mêmes, c'est-à-dire reconnues dans leur principe. C'est bien ce qui est en cause, par exemple, dans l'adhésion aux croyances des personnages, la "vraisemblabilisation" de leurs paroles et de leurs actes et l'admissibilité d'un ordre du monde, comme peut l'indiquer une lecture de Trente arpents.

Une deuxième attitude, tout aussi fondamentale, est transitive. Elle recherche la lisibilité du texte littéraire dans ses applications. Ainsi, les valeurs différenciées d'un point de vue littéral deviennent-elles des schèmes culturels ou littéraires, tels que les genres, les modèles langagiers ou esthétiques. Le modèle du "roman de la terre ", le discours paysan ou encore la bande dessinée comme genre sont de cet ordre. D'un point de vue référentiel, l'attitude transitive fait apparaître l'Histoire, bien entendu, incluant les faits sociaux, politiques ou autres de la réalité extralittéraire. Les épisodes de la Rébellion et de la conscription de même que la place de la connaissance universitaire dans les rapports sociaux y trouvent leur pertinence.

21 Il est certain que la compréhension lexicale doít convoquer la mémoire, mais sans pouvoir s'y réduire. Voir, à ce propos Gilles Thérien, sLecture, cognition, mémoire (ou les cocotiers de Cicéron), dans La recherche littéraire. Objets et métbodes, sous la direction de Claude Duchet et Stéphane Vachon, Montréal, XYZ éditeur, coll. «Théorie et littérature ", 1993, p. 477-486. 
80

Les attitudes analytique et transitive sur des objets littéral et référentiel ne rendent pas compte de toutes les opérations et formes d'investissement du sujet lecteur. Elles ont même l'inconvénient de laisser entendre plutôt un certain détachement. Pour tenir compte de l'investissement entier du lecteur-interprète, il faut poser une autre catégorie correspondant à une transposition dans le domaine affectif des objets de lecture. Dans une perception littérale, l'attitude transpositive retiendra éventuellement un mode d'existence ou un stéréotype mettant en cause, par exemple, la confiance ou la crédulité, la rigueur de la pensée ou l'honnêteté. Cette perception ne conduit pas nécessairement à un programme de comportement, même si l'actualisation des contenus sur un plan personnel en est une conséquence usuelle. En effet, une perception référentielle donnera lieu, le cas échéant, à une identification et à un engagement personnel. Un véritable parti pris de lecture fera ainsi passer l'œuvre dans le champ pragmatique.

\section{La référence personnelle}

En somme, depuis une perception de la dimension littérale et depuis une attitude analytique, la lecture conduit éventuellement à l'action par la transposition des contenus résultant d'une perception référentielle d'un texte littéraire. La référence littéraire devient alors une référence personnelle.

Ce passage de la lettre à l'action, de l'esthétique à l'éthique, représente tout un parcours du sujet-lecteur, et qui est laissé à sa liberté. La référence, dans cet ordre d'idées, n'est qu'une variable recouvrant à son tour un vaste ensemble d'objets perceptibles et interprétables, tels que les schémas d'action, les données historiques et les attentes ou visées personnelles. Si elle est à la fois un phénomène de texte, de co-texte et de contexte, c'est par les possibles transactions qui s'y opèrent entre les attitudes de lecture. Elle apparaît, enfin, au cœur des enjeux de la signification littéraire, quelle que soit la figure de sa légitimité, de l'œuvre à l'institution, et quel que soit l'horizon de son langage, de l'énonciation à la transparence. La lecture cherche ses propres références en (re)construisant celles du texte littéraire. C'est bien parce qu'elle est, aussi, une pensée en action. 